\title{
La batalla de Zacatecas por los distritos regionales innovadores.

\author{
Una opción de desarrollo \\ integral para el estado
}

\author{
Rodolfo García Zamora* \\ Juan Manuel Padilla**
}

Después de 460 años, la economía y la sociedad zacatecanas siguen dependiendo de la explotación de los recursos naturales y la exportación de fuerza trabajo, con una estructura productiva extractivista, polarizada y con gran heterogeneidad económica y social; basada en las ventajas comparativas de la sobre explotación de los recursos naturales, incapaz, crónicamente, de generar los empleos necesarios y provocando la persistencia de la pobreza en 60 por ciento de la población. Ante ello, se plantea la necesidad de construir la nueva institucionalidad del desarrollo de Zacatecas, transitando hacia las ventajas competitivas con cohesión social, basadas en la aplicación de la ciencia y la tecnología, mediante la construcción de los distritos regionales innovadores en las zonas estratégicas de la entidad.

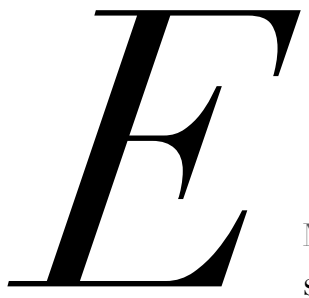

N LAS ÚLTIMAS tres décadas surgen y se han desarrollado en América Latina diversas políticas de desarrollo local, como respuesta de comunidades y territorios a los desafíos de la globalización, a los ajustes macroeconómicos estructurales

* Director de la Unidad Académica de Estudios del Desarrollo de la Universidad Autónoma de Zacatecas, México.

** Docente-investigador de la Unidad Académica de Economía de la Universidad Autónoma de Zacatecas, México. del nuevo modelo de apertura y la mayor competencia de las economías nacionales, a la heterogeneidad productiva, desigualdad social y la pobreza. Pese a compartir los objetivos de aumento de la productividad, el crecimiento y el desarrollo económico, las estrategias de desarrollo local y las políticas estructurales acometen de manera diferenciada el proceso de reestructuración económica y la crisis mundial actual: "Las políticas estructurales adoptan una aproximación funcional, a diferencia de las políticas de desarrollo local, que definen sus acciones con un enfoque territorial". 


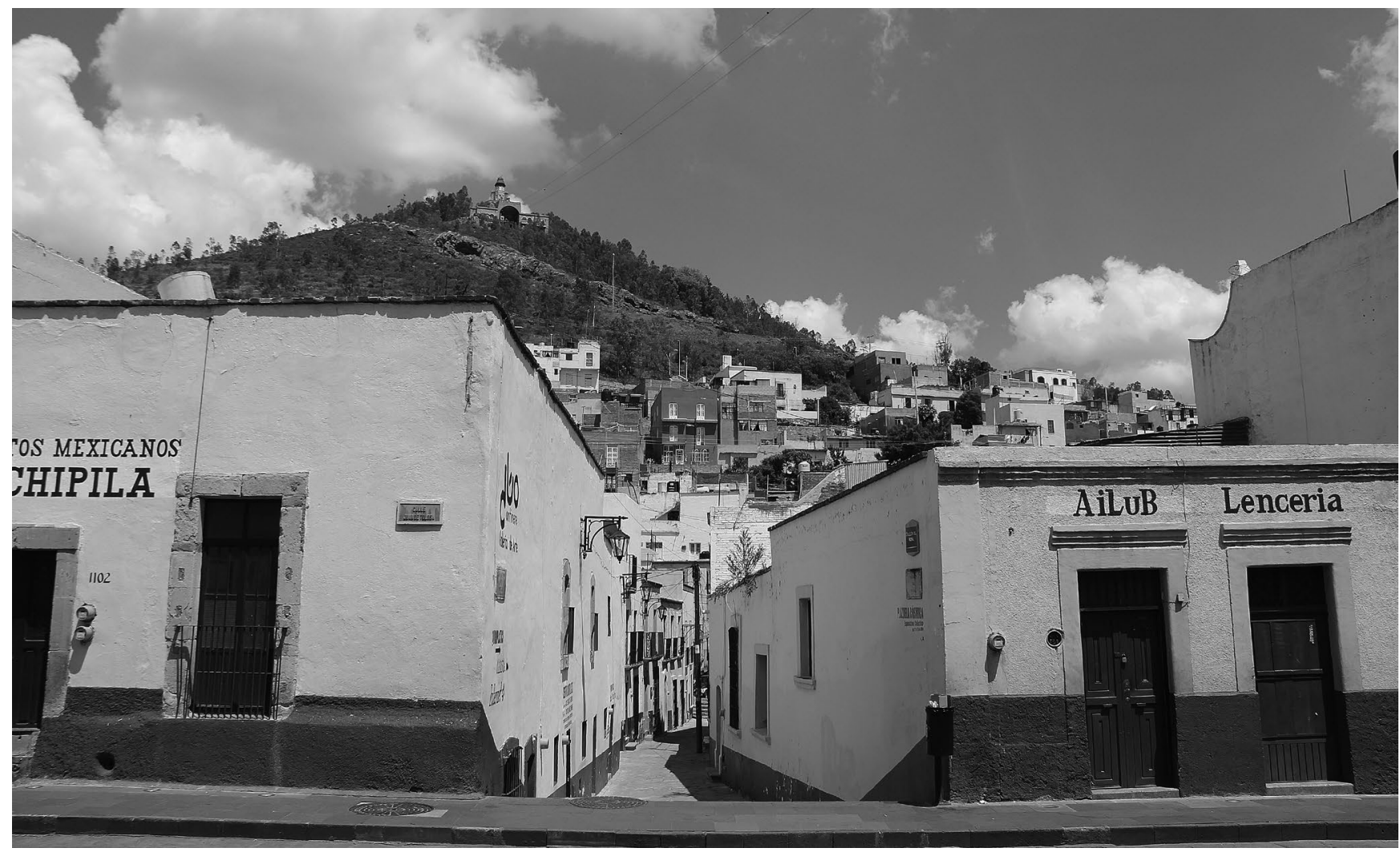

El perfil económico de las localidades zacatecanas es tradicional. Los principales centros poblacionales han sido invadidos por corporaciones nacionales y extranjeras y determinadas áreas rurales tomadas por capitales extractivos. No se ha implementado una estrategia para el desarrollo económico local. Foto: Нunberto Márquez

Al abordar el sistema productivo es necesario tomar en cuenta que "las acciones (procesos económicos) se realizan en territorios caracterizados por un sistema social, institucional y cultural con el que interactúan. Por ello, las medidas son más eficaces cuando utilizan los recursos locales y se articulan con las decisiones de inversión de los actores locales". ${ }^{2}$ En este sentido, los programas de desarrollo humano de la Organización de las Naciones Unidas (ONU) destacan que la valorización de las capacidades locales y la formación de redes territoriales (de empresas, instituciones y entidades de servicios) permiten mejorar la productividad y competitividad de las empresas y territorios. ${ }^{3}$

Bajo la perspectiva anterior, dos factores determinan los resultados del proceso económico: "el potencial de desarrollo existente en cada territorio y la capacidad de organización de los actores locales. Desde este enfoque, todas las localidades y territorios disponen de un conjunto de recursos, que constituyen su potencial de desarrollo, tanto en el caso de las áreas rurales $[\ldots]$ como en las ciudades $[\ldots]$. A nivel de cada localidad, país o territorio se detecta, por ejemplo, la dotación de una determinada estructura productiva, mercado de trabajo, conocimientos técnicos, capacidad empresarial, recursos naturales, estructura social y política, o tradición y cultura, sobre los que necesariamente se articulan las iniciativas locales". ${ }^{4}$ Aunado a esta estructura holística, es fundamental que "los actores públicos y privados ejecuten sus programas de inversión de forma coordinada." Lo que se puede efectuar mediante el manejo de la metodología de Sistemas Productivos Locales, ${ }^{6}$ una versión holística actual de las metodologías de encadenamientos productivos y sistemas productivos.

La rica experiencia de las políticas de desarrollo local en América Latina, específicamente de México, muestra que no hay que olvidar la diversidad estructural entre países y regiones dentro de un mismo país, incluso de un estado como Zacatecas. En efecto, "las necesidades y demandas de las localidades y territorios son diferentes, las capacidades de los habitantes, empresas y comunidades locales cambian, y, además, cada comunidad visualiza de forma diferente las prioridades que deben estar contenidas en las diferentes políticas de desarrollo".? 
Crisis e innovación PRODUCTIVA TERRITORIAL

Luego de cuatro años del estallido de la crisis financiera estadounidense y de múltiples debates y reuniones de organismos como el Fondo Monetario Internacional (FMI), el Banco Mundial (BM), la Organización para la Cooperación y el Desarrollo Económicos (OCDE), entre otros, los impactos siguen afectando severamente a todos los países, como se ratifica en la reciente reunión del Foro Económico de Davos, Suiza, donde el consenso es que prevalece una gran inestabilidad e incertidumbre sobre el futuro de la economía mundial y que resulta imprescindible un marco regulatorio riguroso de las actividades financieras en todo el orbe y un papel activo de las políticas públicas para superar la recesión económica internacional y promover la recuperación y el crecimiento económico.

La profunda crisis económica mundial actual, que de 2007 a 20I4 ha sacudido las estructuras financieras, productivas, sociales, políticas y culturales de todos los países, también tiene el carácter de oportunidad de los sistemas productivos regionales, "de tal manera que se fortalezcan y sean más competitivos a nivel internacional y se conviertan en una salida proactiva a la crisis misma. El elemento clave lo representa la introducción y difusión de las innovaciones en el sistema productivo, social e institucional, ya que es la forma más eficaz de asegurar, a largo plazo, el aumento de la productividad y competitividad". 8

Bajo esta perspectiva, y con un nuevo enfoque analítico de la actividad económica empresarial que supere la visión tradicional del sector, para asumir el enfoque de encadenamientos productivos regionales o clúster $^{9}$ y de distritos de innovación, ${ }^{10}$ el desarrollo económico territorial puede ser una salida proactiva a la crisis económica en curso. Ello significa ir más allá de la actitud reactiva de defenderse de sus consecuencias buscando soluciones concretas a los problemas específicos de los territorios, usando el potencial de desarrollo que no han podido usar a causa de la crisis. Mediante "una estrategia que estimule los rendimientos crecientes de las inversiones y, por lo tanto, propicie el aumento de la productividad y la competitividad de las economías" nacionales y estatales.

Esta perspectiva territorial, de una salida de la crisis económica desde la visión de los actores locales interinstitucionales, busca el progreso social (mayor cohesión) y el desarrollo sostenible. Se entiende, pues, que el desarrollo económico y social es un proceso de construcción social entre los actores en el cual el cre-

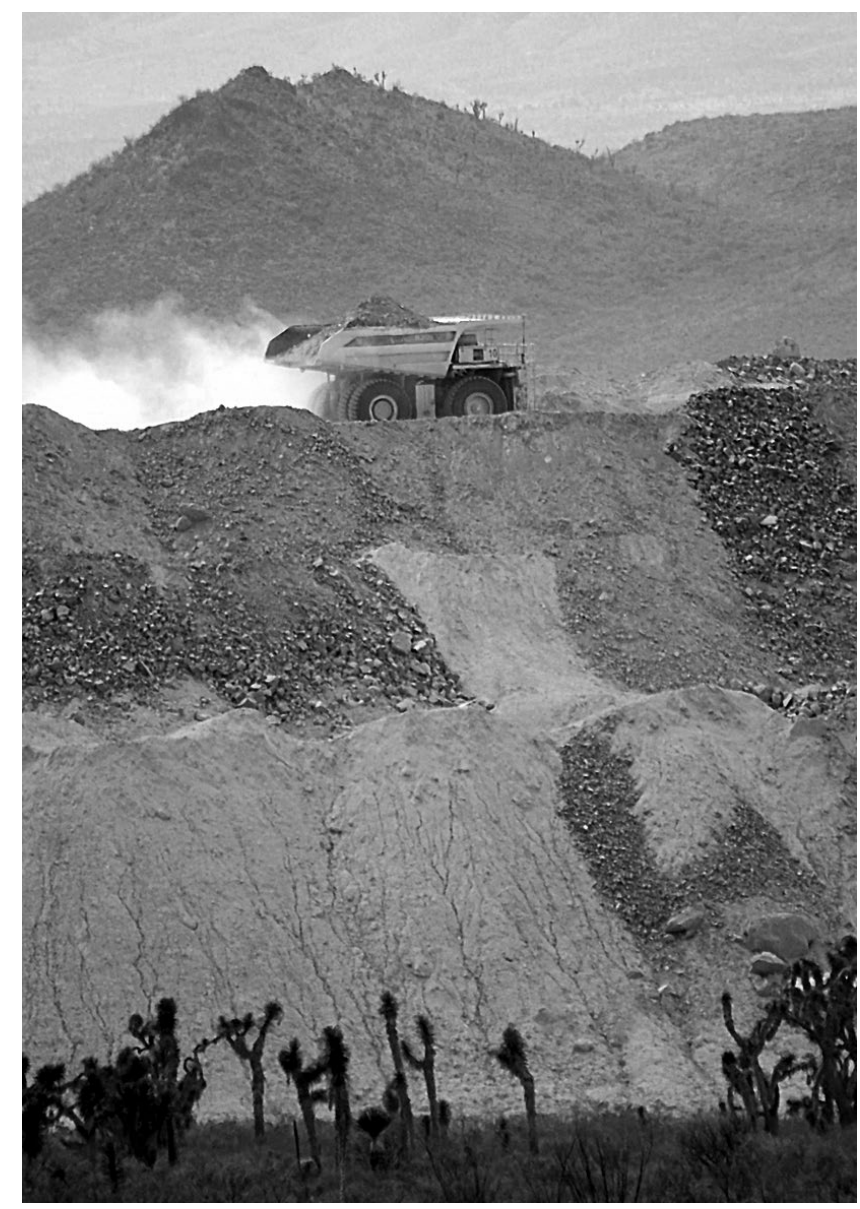

La minería extractiva se ha consolidado como uno de los sectores más dinámicos para el asentamiento de inversión extranjera directa, sin embargo la capacidad de generación de empleo es limitada y los efectos socioamentales son gravosos. Foto: Alfredo Valadez.

cimiento económico y la distribución del ingreso son dos caras de un mismo fenómeno, ya que cuando los actores públicos y privados deciden aplicar sus diferentes recursos lo hacen con la finalidad de aumentar la productividad y mejorar el bienestar de la sociedad.

Las políticas de desarrollo local enfrentan la cuestión del ajuste y de la restructuración de los sistemas productivos en función de la competitividad de las empresas en un marco de competitividad territorial. El crecimiento de la renta y los fuertes cambios en la demanda han provocado la diversificación de la producción tanto en las ciudades como en las áreas rurales. El desarrollo de la actividad turística $[. .$.$] muestra$ cómo los cambios en la demanda internacional estimulan la diversificación productiva y plantean la necesidad de introducir continuamente innovaciones que fortalezcan la competitividad. 


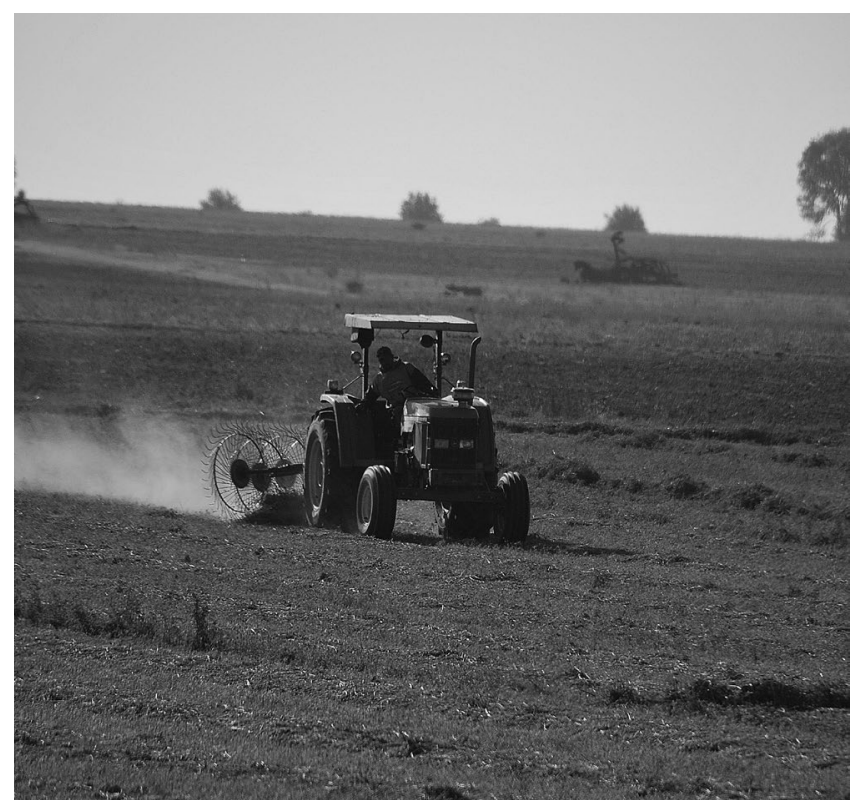

La mayor parte de los productores agrícolas zacatecanos son minifundistas con baja mecanización. Además, padecen el acoso de acaparadores que absorben los mayores márgenes de beneficio.

Foto: Jorge VÁzQuez.

A medida que aumenta la integración económica, las empresas y los territorios desarrollan sus ventajas competitivas en los mercados locales e internacionales. Así, los sistemas productivos están siempre evolucionando y, con frecuencia, la dinamización de los cambios se realiza a partir de la renovación del saberhacer tradicional con la introducción de nuevos conocimientos durante el proceso de cambio estructural."2

Existen experiencias internacionales y nacionales, en localidades y territorios, donde el reto no radica "tanto en la diferenciación de la producción o la reducción de costes como en encontrar nuevos productos para mercados en los que las empresas locales pueden mantener sus ventajas competitivas". ${ }^{33}$ Ejemplo de ello lo representan los cafeticultores de Tapachula, Chiapas, quienes ante la imposibilidad de competir con los productores vietnamitas, tuvieron que reorientarse hacia la producción de flores tropicales para el mercado estadounidense..$^{14}$

Otro ejemplo de cómo las empresas y territorios en México se reorientan hacia la producción de nuevos bienes y servicios, cuya demanda crece en los mercados internacionales, así como los productos que incorporan alta tecnología, es el clúster de la electrónica en Jalisco. La clave para que estas transformaciones sean posibles radica en la mejora constante de los recursos humanos y la introducción de innovaciones de productos y procesos en el tejido productivo territorial. ${ }^{5}$ En este sentido, la ovu le asigna una gran prioridad a la formación de recursos humanos en los programas de desarrollo humano, con lo que estimulan las capacidades de la población local y dinamizan el entorno en el que surgen las iniciativas. Además, en muchos países como Ecuador, Colombia, Cuba, Uruguay, Guatemala, Marruecos y otros, se han puesto en marcha programas de formación de agentes de desarrollo local $(\text { ADL })^{16}$ mediante los cuales se capacita a las personas para que sean capaces de gestionar iniciativas locales, proyectos empresariales y de desarrollo local con un enfoque integral.

Como lo muestran múltiples experiencias de la Unión Europea en países como Irlanda, Portugal, Grecia y España, que en el pasado mostraban menores niveles de desarrollo: "El desarrollo local es, también, una estrategia que se basa en la mejora continua de los recursos disponibles y, particularmente, de los recursos naturales y del patrimonio histórico cultural, ya que con ello se contribuye a aumentar la ventaja competitiva del territorio y el bienestar de la población" ${ }^{77}$

Una estrategia de desarrollo económico territorial o desarrollo local integral requiere, especialmente, la participación de todos los actores y las redes territoriales en el diagnóstico, diseño, ejecución y control de las acciones y proyectos de desarrollo. Este tipo de estrategias cuentan ahora, afortunadamente, con aliados institucionales de carácter mundial muy importantes como el Programa de las Naciones Unidas para el Desarrollo (PNUD), la Organización Internacional del Trabajo (OIT), la OCDE y la Comisión Económica para América Latina y el Caribe (Cepal), entre otros.

\section{ZACATECAS: RETO DE COMPETITIVIDAD, INNOVACIÓN Y COHESIÓN SOCIAL}

El estado de Zacatecas se ha caracterizado desde fines del siglo XIX por lo atrasado de su estructura económica y por la incapacidad para generar los empleos necesarios que ha requerido la población para acceder a una vida digna. Esto explica, en gran medida, el constante y creciente éxodo migratorio zacatecano a otros estados de México y hacia Estados Unidos, que si bien ha significado algunos impactos positivos, como la llegada creciente de remesas hasta antes de la crisis de 2007 y la existencia de más de 200 clubes de migrantes 


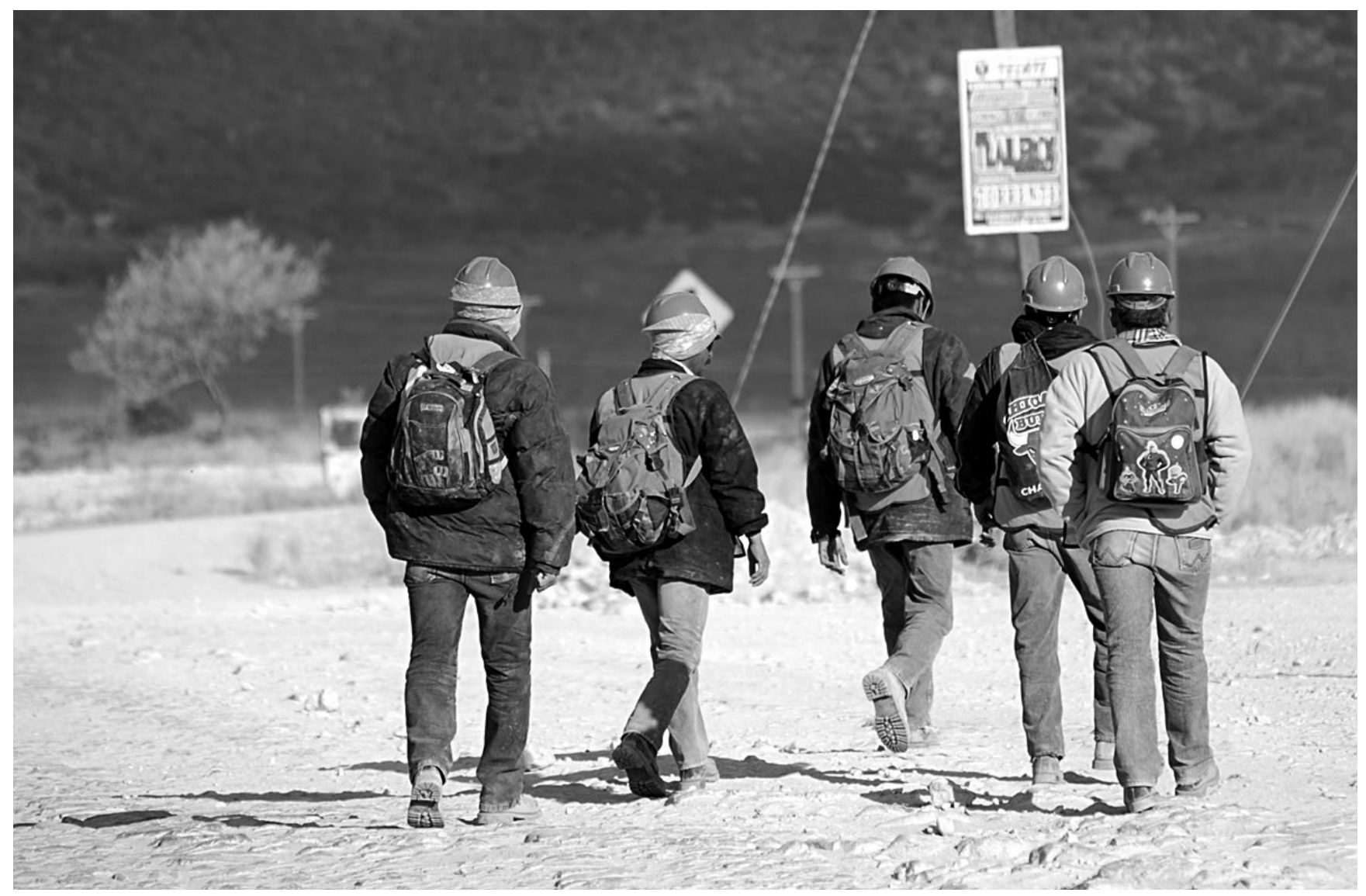

Los trabajadores de las minas cubren jornadas extenuantes, afrontan enfermedades y accidentes, además de que perciben bajas remuneraciones.

Foto: Alfredo Valadez.

en aquel país que han financiado, desde r 993 hasta la fecha, más de 5,ooo proyectos de infraestructura básica mediante el Programa 3xi, por otra parte ha profundizado la tendencia hacia el despoblamiento en la mayoría de los municipios, volviendo imperiosa la promoción del desarrollo regional integral.

Zacatecas, como muchos otros estados mexicanos y regiones de América Latina, sigue caracterizándose por sus rasgos de atraso económico, baja productividad, bajo nivel de ingreso, baja contribución al producto interno bruto (PIB) nacional, desigualdad social y la prevalencia de la pobreza en un porcentaje importante de la población estatal, como lo muestran los siguientes indicadores del Plan Estatal de Desarrollo 2011-2016: el producto interno bruto (PIB) estatal en promedio fue de 65 mil millones de pesos entre 2005 y 2010 , cantidad que representa el o.8 por ciento del PIB nacional para el periodo. El PIB per cápita de 2008 era de 46 mil pesos, equivalente al 59 por ciento del indicador nacional. En el mismo año, aportaba r.I9 por ciento a la población ocupada del país. La productividad promedio por hombre ocupado es 33.5 por ciento, por debajo del nivel nacional. ${ }^{18}$ En cuanto a la composición sectorial del PIB estatal respecto al nacional destaca el aporte del sector primario del II por ciento respecto a 4 por ciento a nivel nacional, frente a un aporte similar del sector secundario de 28 y 32 por ciento y del sector terciario de 6 r y 64 por ciento, respectivamente. ${ }^{19}$ Esta situación económica explica los limitados avances en términos de desarrollo humano de la población estatal (vida prolongada, acceso a la educación, salud y goce de una vida digna) que hace que nuestra entidad se ubique en el lugar 26 del país respecto al índice de desarrollo humano. ${ }^{20}$ Uno de los aspectos preocupantes en este sentido lo representa la situación de la pobreza estatal, ya que “20.9 por ciento de la población padece pobreza alimentaria, el 29.3 por ciento pobreza de capacidades, y el 53.6 por ciento de patrimonio"; ; indicadores que están por encima de la media nacional. "Zacatecas ocupa el lugar i 3 en orden descendente en cuanto al índice de marginación, [...] (un) grado de marginación medio; sin embargo, nueve de sus municipios son considerados como de alta marginación". ${ }^{22}$ 
"Si se considera de forma agregada, el 52 por ciento de las personas en Zacatecas viven en pobreza multidimensional, esto es, que padecen carencias en cuanto a su bienestar económico, como el acceso a los satisfactores que por derecho les corresponden para su desarrollo social (salud, seguridad social y vivienda). A nivel nacional, la cifra es de 44.2 por ciento". ${ }^{23}$ En el mismo Plan Estatal de Desarrollo 2011-2016 se reconoce la existencia de profundas brechas sociales entre los diferentes integrantes de la sociedad a nivel de ingreso, región y género.

Como indicamos antes, el atraso económico estatal explica la larga tradición migratoria nacional e internacional de Zacatecas, lo que ha propiciado que pese a que la entidad presenta similares tasas de natalidad y mortandad a las existentes a nivel nacional, su crecimiento demográfico ha sido mucho menor, al grado de que entre $199^{\circ}$ y 2005 , prácticamente se estancó, a tal punto que, para 2005, 43 de los 58 municipios mostraron una reducción de su población respecto a 2000. ${ }^{24}$ Esta tendencia de una baja persistente en la población estatal y de despoblamiento en varias de sus regiones se rompe como resultado, en gran medida, de la crisis económica en Estados Unidos, el aumento en el desempleo (I7 por ciento para latinos), la creciente militarización de la frontera norte y la xenofobia generalizada contra los migrantes indocumentados en el país vecino que provocan una caída radical en la migración internacional hacia Estados Unidos. Todos los elementos anteriores han propiciado que la población estatal crezca de I'367,692 habitantes en 2005 a I'490,55o habitantes en 2010.

En el último quinquenio, la entidad experimentó una tasa de crecimiento demográfico de ı.9 por ciento, cifra no vista durante los últimos cincuenta años. Se trata de 122 mil 448 personas más que incrementan los retos en materia de empleo, educación y salud, principalmente. ${ }^{25}$ Así, 52 de los 58 municipios presentan tasas positivas de crecimiento poblacional como resultado, en gran medida, del retorno de un número importante de migrantes zacatecanos, por la ausencia de empleos en los últimos cuatro años en el país vecino, el regreso de un número creciente de ellos y la deportación de otro número significativo. Nuevo fenómeno económico y social que incrementa la necesidad de generar empleos en la entidad y la generación de nuevas políticas de desarrollo local integral.

Dentro de la dinámica demográfica estatal destaca la reducción de la población urbana de 5o.6 por ciento en 2005 a 48.2 por ciento en 2010 y la concentración de la población y de la actividad económica en los mu-

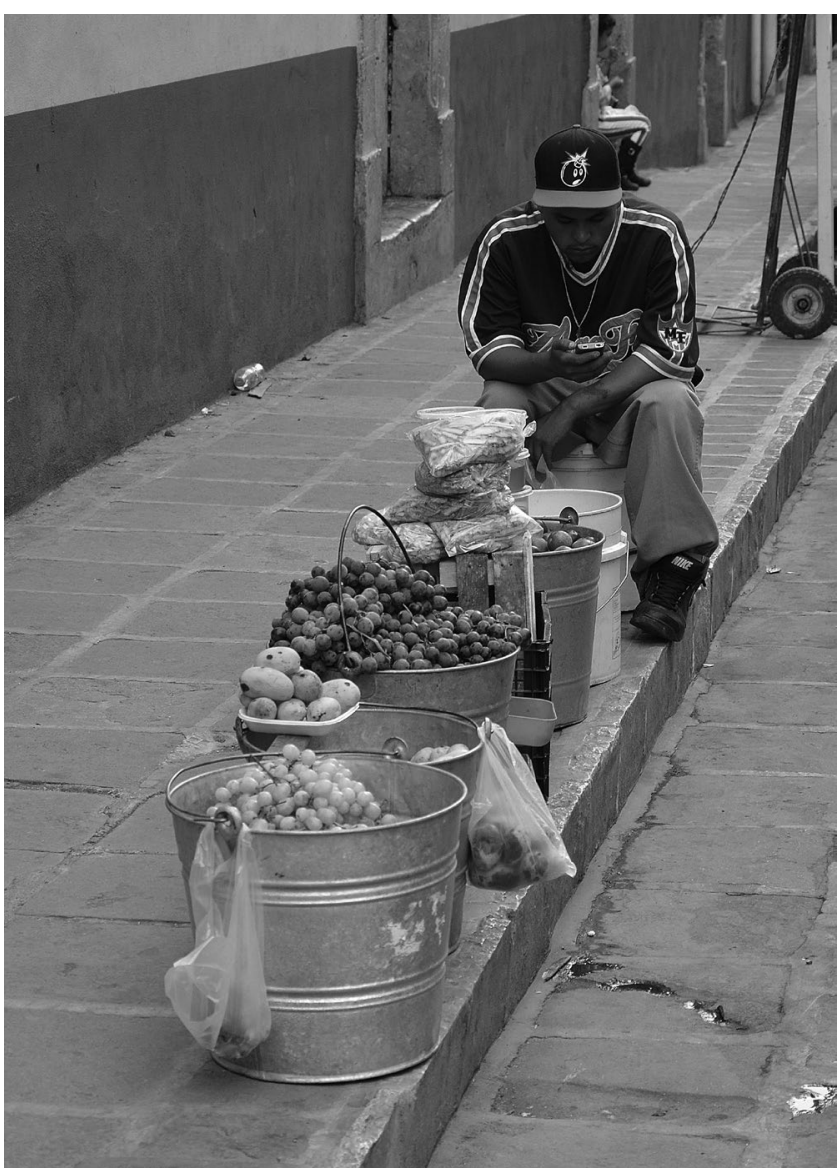

Vendedor ambulante de productos regionales, frutos y nopales, en el centro de la ciudad de Zacatecas. Foto: Jorge VázQuez.

nicipios de Zacatecas, Guadalupe y Fresnillo, que, considerando Calera, comprende 27 por ciento de la población estatal, conforman la llamada zona metropolitana que reclama con urgencia el diseño y aplicación de políticas integrales de desarrollo urbano y políticas de convergencia económica y social regional que reduzcan la brecha entre estos municipios y el resto de la entidad.

Frágil estructura económica estatal

El PED reconoce que: "Durante el periodo 2003-2009, la economía de Zacatecas registró un crecimiento promedio anual en términos reales de 3.9 por ciento, presentándose incrementos sostenidos en el PIB de la mayoría de las ramas económicas. Sin embargo, este crecimiento ha sido insuficiente para generar los más de ocho mil empleos anuales que requiere Zacatecas", ${ }^{26}$ pero sobre todo ha sido muy escaso para mejorar el nivel de ingreso y de vida de sus ciudadanos. 
En gran medida, el atraso económico de Zacatecas se explica por su función tradicional de proveedor de materias primas y mano de obra para la industrialización de otras regiones del país y el extranjero tanto al inicio del siglo xx como durante la etapa de industrialización por sustitución de importaciones de 1940 a 1982. Así, la industria estatal es muy reducida, los cinco parques industriales tienen un lento funcionamiento y raquítico impacto regional con sólo 83 establecimientos. La inversión extranjera, si bien se ha incrementado de I5.4 millones de dólares en 2006 a ı, 506 millones en $2008,{ }^{27}$ sigue siendo una inversión con carácter de enclave, concentrada en la minería, con el mismo enfoque extractivista depredador de los recursos naturales, del medio ambiente y de la mano de obra que en el resto del país y de América Latina, con escasos eslabonamientos e impactos económicos regionales.

La débil inserción de Zacatecas en la economía mundial mediante el comercio exterior refleja lo débil de la estructura económica, la baja competitividad y la enorme dependencia de la exportación de minerales y mano de obra. En efecto, las exportaciones estatales en 2008 ascendieron a 703 millones de dólares, que representaban 0.24 por ciento de las exportaciones nacionales; en 2009 suben a r,o74 millones de dólares, que representaron 0.46 por ciento del total nacional. Respecto al aporte del sector exportador al PIB estatal, en 2009 fue de I/4 por ciento, mientras a nivel nacional fue de 26 por ciento ${ }^{28}$.

Como en la inversión extranjera, "el tamaño de las exportaciones de mercancías reflejan que Zacatecas tiene un bajo nivel de integración a la economía mundial, lo que se convierte en una importante desventaja en los esfuerzos por acelerar el crecimiento económico del estado y fortalecer su desarrollo humano". ${ }^{29}$ Entre los destinos de exportaciones más importantes se encuentran Estados Unidos, Canadá, Bélgica, Italia, Suiza, España, Puerto Rico y Japón. Los principales productos exportados son la cerveza, papel, cartón, autopartes, prendas de vestir, concentrados de zinc, productos cerámicos, carne, legumbres y hortalizas. ${ }^{30}$

A su vez, la frágil estructura económica estatal explica la baja capacidad de generación de empleos formales de cuatro mil en los mejores años, cuando el estado requiere por lo menos de io mil nuevos empleos cada año para atenuar el fuerte flujo migratorio estatal que antes de la crisis de 2007 era de 30 mil habitantes al año, el 5o por ciento de los cuales emigraba a Estados Unidos. Es importante destacar que, al igual que a nivel nacional, el sector terciario es el más dinámico en la generación de empleos, contribuyendo con 53.7 por ciento $(29 \mathrm{I}, 25 \mathrm{I})$, seguido del sector primario con 27.7 por ciento $(150,357)$ y el sector secundario con I8.2 por ciento $\left(9^{8}, 450\right)$.

Los datos de la actividad minera en el estado son contradictorios, ya que, como indicamos antes, si bien la inversión extranjera ha crecido de forma significativa su impacto en el desarrollo económico regional y estatal es muy reducido. La producción de plata y plomo representan 46 y 35 por ciento de la producción nacional, respectivamente. A nivel estatal, Fresnillo aporta zo por ciento de la producción de plata y 23 por ciento de la producción de plomo; Mazapil aporta 32 por ciento de la producción de zinc y Sombrerete 25 por ciento de la producción de cobre. ${ }^{31}$

El sector agropecuario estatal, al igual que a nivel nacional, sigue teniendo una gran importancia económica, social y política en la entidad. La agricultura se desarrolla en, aproximadamente, I.3 millones de hectáreas, que representan casi 6 por ciento de la superficie cultivada del país. Esa actividad genera una producción cuyo valor actualmente es poco más de io mil millones de pesos. ${ }^{32}$ La principal limitación natural para el avance de la agricultura estatal es la dependencia climatológica y la reducida infraestructura hidráulica, ya que sólo i2.7 por ciento de la superficie cultivada en el estado cuenta con riego. La producción estatal se concentra en los granos básicos, especialmente frijol (39 por ciento) y maíz (23 por ciento), seguida de la avena forrajera, el trigo y el chile verde. ${ }^{33}$

En el contexto nacional, el sector agropecuario zacatecano hace las siguientes aportaciones: en frijol aporta 25 por ciento de la producción nacional; en avena forrajera, el 20 por ciento; en chile verde, el ro por ciento; en tomate verde, el 7.8 por ciento; en carne en canal, el 7.4 por ciento, y en cera en greña, el 6 por ciento. ${ }^{34}$

Pese a los indicadores anteriores, la productividad agrícola estatal sigue siendo muy baja comparada con los promedios nacionales a causa de las limitaciones climatológicas, lo limitado de las políticas públicas de desarrollo rural, la reducción del extensionismo agrícola, la ausencia de planeación y capacitación agrícola, una infraestructura insuficiente y el rezago tecnológico. Ejemplo de ello es el frijol, cuyo rendimiento en 2008 fue un 22.9 por ciento, inferior con respecto a la media del país; en el caso del maíz fue 5o.9 por ciento menor y en el chile verde 54.9 por ciento. Los cultivos donde los rendimientos son mayores a la media nacional son alfalfa, pastos y tomate verde..$^{3 \check{5}}$ 
En general, la estructura agropecuaria sigue basada en una economía campesina tradicional, de baja productividad y competitividad, rezagada tecnológicamente, con graves problemas de comercialización y trabas para transitar al desarrollo agroindustrial, prueba de ello son los sistemas tuna-nopal, durazno, vid, guayaba, ganado vacuno, y últimamente agave, que desde hace por los menos cuatro décadas han tenido un gran potencial de desarrollo agroindustrial, y, en general, siguen siendo actividades limitadas a la simple producción y venta a intermediarios de otros lugares de México sin ningún valor agregado. Este raquítico sector agropecuario ha sobrevivido en Zacatecas por el efecto compensador de dos factores: el pluriempleo familiar y la emigración. Ambos se han erosionado gravemente por la crisis económica estructural del país, la falta crónica de empleo y la caída de la migración internacional y de las remesas. En este sector urge el diseño de nuevas políticas públicas de desarrollo rural que posibiliten transitar de la estructura productiva tradicional, a los encadenamientos productivos regionales (clúster) y los distritos territoriales innovadores.

En cuanto al sector del turismo, esta es una actividad señalada por los últimos tres gobiernos como una estrategia importante para el desarrollo de la entidad, aprovechando la riqueza histórica de la capital, Guadalupe, Fresnillo, Jerez y otros lugares, que si bien ha experimentado un aumento en la llegada de turistas, que en 2010 ascendió a 422,535 visitantes, con un gasto promedio 677 pesos diarios en la zona Zacatecas-Guadalupe ${ }^{36}$ sigue presentando la limitación de una oferta integral de servicios turísticos que aumente el flujo de visitantes y permita una estancia mayor al promedio de los últimos años de r.5 días por visitante.

Finalmente, respecto a la industria manufacturera, de acuerdo al "Sistema de Cuentas Nacionales de México, esta industria genera en Zacatecas un PIB de 12.3 mil millones de pesos a precios corrientes, que representa 0.62 por ciento del PIB manufacturero nacional, lo que ubica a Zacatecas como la séptima entidad federativa con menor actividad manufacturera. Entre 1995 y 2009, las manufacturas estatales crecieron a un $3.3 \%$ por ciento promedio anual en términos reales". ${ }^{37}$ En la economía estatal, "la manufactura aporta el I2.2 por ciento del PIB estatal, lo que la ubica por arriba del sector agropecuario, pero por debajo de la minería, el comercio y los servicios inmobiliarios. Por rama de actividad predomina ampliamente la industria de alimentos, bebidas y tabaco, que aporta el 83 por ciento del PIB manufacturero estatal. Le

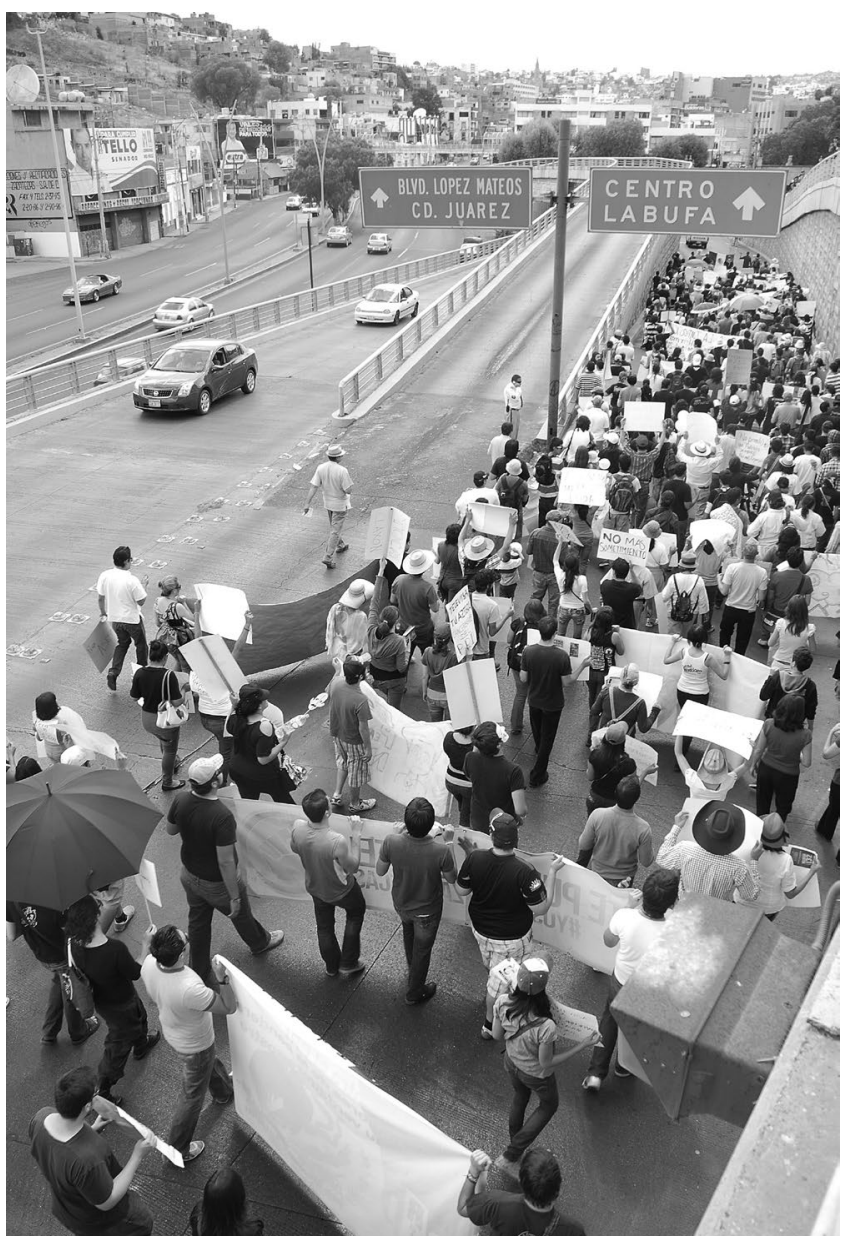

$$
\begin{gathered}
\text { Marcha de protesta de organizaciones sociales } \\
\text { por las vialidades de la ciudad de Zacatecas. } \\
\text { Foto: Jorge VázQuez. }
\end{gathered}
$$

siguen las industrias metálicas con un 6.9 por ciento y la fabricación de productos a base de minerales no metálicos con el 3.I por ciento". ${ }^{38}$

Acorde a los Censos Económicos de 2009, "la industria manufacturera zacatecana está compuesta por 4,969 unidades económicas, representando I.I4 por ciento de las unidades económicas a nivel nacional, empleando a 29,677 personas en la entidad". ${ }^{39}$ Por indicadores de tamaño y productividad de las empresas manufactureras zacatecanas, éstas están en la mayoría de los casos por debajo con respecto de los promedios nacionales (PED 2OII-20I6):40

En cuanto al tamaño promedio medido por la ocupación, en Zacatecas es de apenas 5.9 personas por unidad, un 42.4 por ciento menor que la media nacional de io.3 personas. La remuneración media por trabajador es un 40 por ciento menor en la entidad. Los ingresos medios por unidad económica, son 52.7 por 
ciento más bajos para las empresas ubicadas en Zacatecas. El ingreso medio generado por persona ocupada, que es un indicador de la productividad de la fuerza de trabajo, es un 17.9 por ciento inferior en la entidad respecto del indicador para el país.

Un elemento adicional a considerar es que 99.5 por ciento son micro, pequeñas y medianas empresas caracterizadas por su atraso tecnológico, baja productividad y reducida capacidad de competencia en el mercado nacional e internacional. Situación que para ser revertida requiere de una estrategia que asuma las fortalezas y debilidades de estos sistemas productivos tradicionales y genere los planes, programas y proyectos que faciliten el tránsito hacia los encadenamientos productivos regionales y la creación de los distritos territoriales de innovación.

Tres rasgos fundamentales caracterizan la estructura económica y empresarial del estado de Zacatecas y condicionan su desarrollo futuro: el rezago económico estructural respecto al resto del país y la economía mundial, expresado en la persistencia de actividades tradicionales, servicios no cualificados y una baja productividad y competitividad. La heterogeneidad productiva sectorial donde sólo o.5 por ciento de las empresas manufactureras, mineras y agrícolas rebasan el perfil de Pymes y son capaces de competir eficazmente en la economía nacional e internacional. El resto, se ubica como micro empresas, Pymes, y unidades campesinas tradicionales, que luchan desesperadamente por sobrevivir frente a la apertura total del mercado nacional, la férrea competencia mundial, la ausencia de políticas públicas consistentes para esos sectores y la ausencia del sistema financiero como soporte de transformación de su forma caduca de funcionamiento. Y por último las crecientes brechas espaciales, tecnológicas, productivas y sociales entre la emergente zona metropolitana de ZacatecasGuadalupe-Calera-Fresnillo, que concentra 27 por ciento de la población y 6i por ciento del PIB estatal y el resto de los 58 municipios de la entidad.

A pesar de que en el Plan Estatal de Desarrollo Zacatecas 20II-20I6 no se reconocen las tres limitaciones estructurales anteriores, sí se ubica como el eje de la política económica de desarrollo la generación de empleo, visualizando a éste como el medio principal del ser humano para acceder a una vida digna y de contribuir al mejoramiento de su sociedad. Por ello, se considera como "prioridad y principal reto la generación de 8 mil nuevos empleos que anualmente se re- quieren para absorber a las y los zacatecanos que se incorporan a la población económicamente activa, más los empleos adicionales necesarios para absorber a los acumulados de años anteriores". ${ }^{4}$

Bajo la perspectiva anterior, el PED 2OI-20I6 destaca que para hacer frente a los retos que demanda el desarrollo económico,

[... nuestro Estado debe insertarse eficazmente en la Economía Global, posicionándose dentro de los flujos mundiales de comercio, inversión, tecnologías y conocimientos. Lo anterior podrá lograrse en la medida en que se fortalezca su competitividad, mediante la consolidación de sus relaciones económicas con los estados vecinos, derivando en una auténtica integración regional. De igual forma, dado el bajo desempeño económico de la mayoría de nuestras actividades productivas, nuestro estado presenta el doble reto de modernizar e incrementar la productividad de sus sectores tradicionales como la agricultura, la ganadería, la minería y el turismo, así como incentivar el desarrollo de nuevos sectores dinámicos en la industria y los servicios, relacionados con las tendencias dominantes de la economía internacional. ${ }^{42}$

Todo lo anterior, expresado en el PED 2OII-20I6, sólo se podrá lograr promoviendo una estrategia de fortalecimiento de la estructura empresarial estatal que permita transitar de la estructura empresarial tradicional existente hacia los encadenamientos productivos sectoriales ("clusters") y la constitución progresiva de los distritos territoriales innovadores junto con el resto de los actores económicos y sociales de las diferentes regiones del estado de Zacatecas, incrementando la competitividad sistémica regional: competitividad económica, cohesión social y sustentabilidad ambiental.

\section{Conclusiones}

Derivado de lo anterior, es necesario realizar las siguientes acciones para construir la competitividad sistémica regional: empezar por realizar un diagnóstico actualizado de la estructura empresarial en Zacatecas, detectando las bases materiales actuales de las ventajas comparativas de los sistemas productivos locales y los recursos potenciales que pueden posibilitar el tránsito hacia las ventajas competitivas dinámicas con un alto contenido tecnológico. De ahí será necesario investigar, seleccionar y fortalecer los sectores económicos 


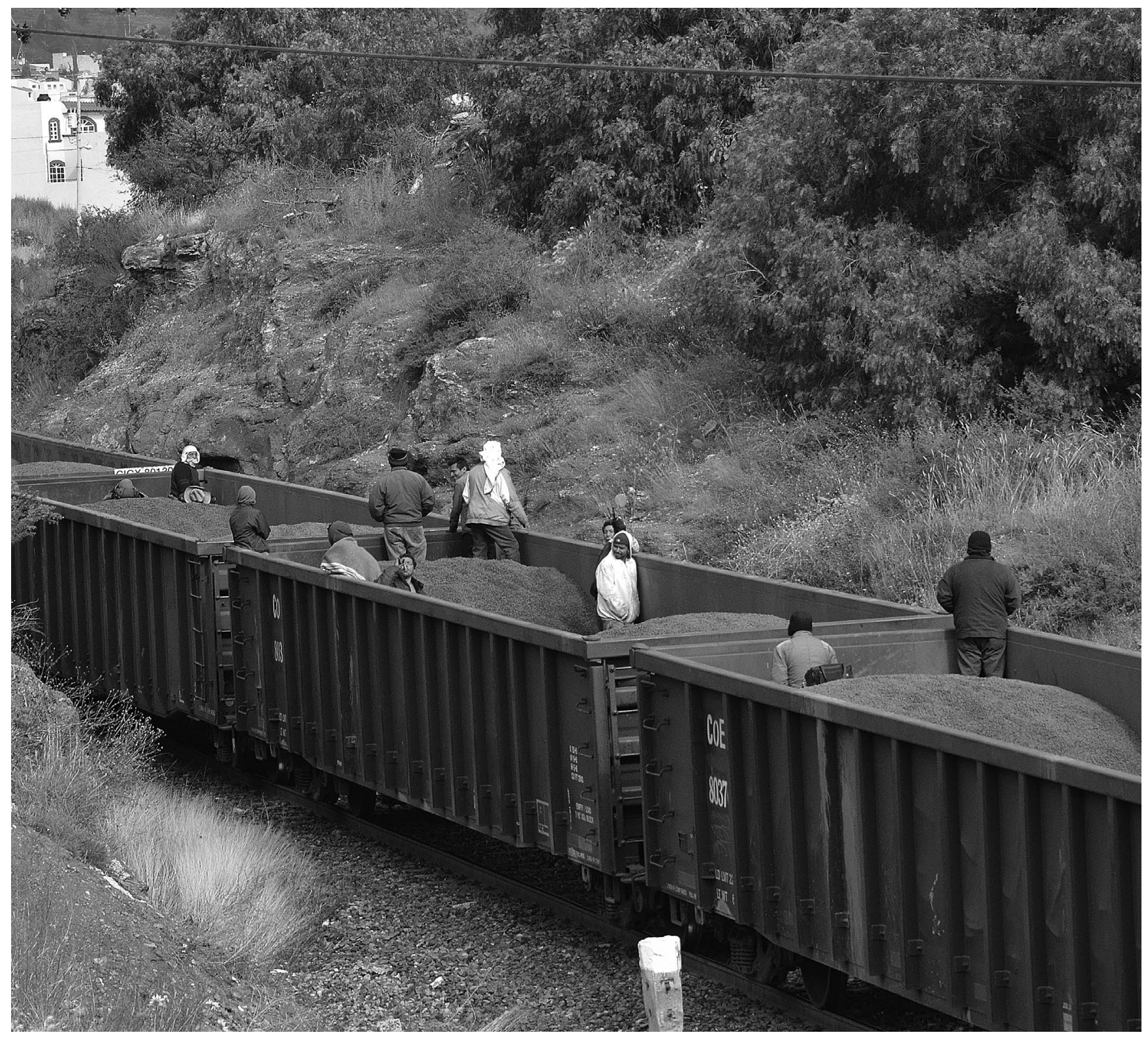

Migrantes indocumentados viajan como polizones en el tren carguero en las inmediaciones de Zacatecas. Foto: Jorge Vázquez.

con mayor potencial de encadenamiento productivo regional en la entidad que pueden actuar como detonantes y soporte del desarrollo local y regional integral, entre los que se encuentran los insumos automotrices, turismo, software, energías alternativas, granos básicos (frijol, maíz), frutales (tuna-nopal, durazno, guayaba), hortalizas (tomate rojo, tomate verde, chile verde) y agave. Construir una propuesta de interrelación de estos sectores "clusterizados" con la estructura social, cultural e institucional de su entorno que permita avanzar hacia la etapa de construcción de los distritos territoriales de innovación. Con los insumos anteriores es de mucha importancia la creación del sistema estatal de ciencia y tecnología, con una agenda estratégica de investigación de los siguientes temas: empleo, pobreza, alimentación, agua, energías alternativas, seguridad humana, nanotecnología, jóvenes, recursos naturales y sustentabilidad y tecnologías de la información y la comunicación. A la par, diseñar una propuesta de colaboración del sistema de educación media, media superior y, particularmente, educación superior del estado con los sectores "clusterizados" y con los que están en vías de conformarse bajo esa nueva modalidad para lograr la colaboración entre las demandas de los sectores productivos más relevantes en las diferentes regiones del estado para generar nuevos 
procesos productivos, nuevos procesos de transformación, de comercialización y venta en el mercado nacional e internacional.

Esta propuesta, además de fortalecer la capacidad de innovación de las empresas estatales, permitirá una modificación de los diferentes planes de estudio desde el nivel medio hasta el posgrado, de manera que posibilite una formación integral, directamente vinculada al mercado de trabajo estatal en proceso, de formación bajo los nuevos criterios impuestos por los criterios de competitividad nacional e internacional que serán el referente del funcionamiento de las empresas zacatecanas. Esta retroalimentación hará viable un programa de vinculación y extensión educativa articulado orgánicamente con las necesidades del desarrollo territorial mediante la innovación permanente para el mejor funcionamiento de las empresas y solución de los problemas locales como urbanización, medio ambiente, energías alternativas, administraciones locales, seguridad humana, cohesión social y sustentabilidad.

También será necesario proponer una estructura organizativa para el inicio de la construcción social por los diversos actores locales, en particular, empresarios, instituciones educativas, tres niveles de gobierno y organizaciones de la sociedad civil, de los distritos territoriales innovadores inicialmente en Zacatecas-Guadalupe, Fresnillo-Valparaíso, Jerez-Monte Escobedo, Río Grande-Sombrerete, Juchipila-Jalpa, TlaltenangoEl Teúl, Loreto-Ojo Caliente.

Esta estrategia requiere del establecimiento de políticas públicas explícitas de desarrollo regional y de empleo en la entidad con el gobierno del estado como principal responsable y promotor de ellos mediante políticas y programas de inversión pública diseñados y operados con consistencia técnica, rigurosidad y transparencia en su aplicación, con un control social y transparencia por parte de todos los sectores productivos y sociales. Esto significa que las administraciones estatales deben dejar de ser simples gestores de los programas federales y administradores de la pobreza de la entidad para diseñar verdaderas políticas econó- micas y sociales de generación de empleo y erradicación de la pobreza como prioridades de toda la administración pública y del presupuesto estatal.

Transitar de las ventajas comparativas a las competitivas significa la reestructuración a fondo de todo el sistema educativo estatal como soporte de esa gran transformación desde la primaria hasta el posgrado para garantizar la formación de ciudadanos integrales a nivel técnico, social y político para que actúen como los actores de cambio en todo el tejido productivo y social de Zacatecas. La educación, la ciencia, la tecnología y la cultura deberán ser la palanca de la gran transformación de Zacatecas.

Esta gran transformación implica construir las bases institucionales del desarrollo competitivo con cohesión social mediante la capacitación permanente de todas las administraciones municipales y de los gobiernos estatal y delegaciones federales por parte de la Universidad Autónoma de Zacatecas (UAZ) con la colaboración del Colegio de México (Colmex), el Centro de Investigación y Docencia Económica (CIDE), El Colegio de la Frontera Norte (Colef), la Comisión Económica para América Latina y el Caribe (Cepal), el Programa de las Naciones Unidas para el Desarrollo (PNUD), la Organización Internacional del Trabajo (OIT) y el Banco Interamericano de Desarrollo (BID) y otros organismos internacionales.

El futuro de Zacatecas sólo será viable con una gran transformación como la antes señalada que garantice empleo, ingresos suficientes, bienestar y democracia. Por ello, esta propuesta de gran transformación no puede ser economicista sino una estrategia integral de seguridad humana que considere la educación, la ciencia, la tecnología, la cultura y el deporte como los soportes del cambio estructural en la producción y la vida social de todo el estado. Erradicando la marginación, la pobreza, la migración como condena, la polarización regional y social mediante un esfuerzo sistemático y transgeneracional de los siguientes 30 años hacia un Zacatecas democrático, equitativo, sustentable y con seguridad humana.

\section{$-\operatorname{notas} \cdot-$}

'Antonio Vázquez Barquero (2009), "Desarrollo local, una estrategia para tiempos de crisis", Universitias Forum, vol. I, núm. 2, p. 4-5.

Ibid., p.5

Ibid., p.6
Idem, p. .5

5Idem.

${ }^{6}$ Francisco Albuquerque (1999), Manual del Agente de Desarrollo Local, Ediciones Sur, Santiago de Chile; Antonio Vázquez Barquero (2009), "Una salida territo- 
rial de la crisis. Lecciones de la experiencia latinoamericana". EURE Revista Latinoamericana de Estudios Urbanos Regionales, vol. xxxv, No. 105 .

ᄀAntonio Vázquez Barquero, op. cit., p. 5.

${ }^{8}$ Idem.

${ }^{9}$ M. Piore, and C. Sabel (1984), The Second Industrial Divide, Nueva York: Basic Books.

${ }^{\circ} \mathrm{G}$. Becattini, (ed.) (1987), Mercato e Forze Locali: il Distretto Iindustriale, Bologna: Il Mulino.

"Vázquez Barquero, op. cit, p. 9.

"Ibid., p.6.

${ }_{13}$ Idem.

IIdem.

"Idem.

${ }^{16}$ Vázquez Barquero, op. cit, p. 8.

"Ibid., p.io.

${ }^{8}$ Gobierno del Estado, (2010), Plan Estatal de Desarrollo, Zacatecas 2010-2016, p. 34.

${ }_{19}$ Idem.

${ }^{20}$ Idem.

"Idem.

${ }^{2}$ Gobierno del Estado, op. cit., p. 36.
${ }^{23}$ Idem.

${ }^{24}$ Gobierno del Estado, op. cit., p. 37.

25Juan Manuel Padilla (2010), "Contexto económico de Zacatecas", WorkingPaper, Unidad Académica de Economía, Universidad Autónoma de Zacatecas, México.

${ }^{26}$ Gobierno del Estado, op. cit.

${ }^{2}$ Ibid., p. 88.

${ }^{28}$ Ibid., p. 89 .

${ }^{29}$ Ibid., p. 9 o.

${ }^{30}$ Idem.

${ }^{3}$ Gobierno del Estado, op. cit., p. 9ז.

32Ibid, p. 92.

${ }^{3}$ Idem.

3itdem.

${ }^{35}$ Gobierno del Estado, op. cit., p. $9^{3}$.

${ }^{36}$ Ibid., p. 94.

3Tlbid., p. 95 .

${ }^{38}$ Idem.

${ }_{3}^{3}$ Idem.

${ }^{40}$ Gobierno del Estado, op. cit., pp. 95-96.

ilbid., p. 87.

估Idem.

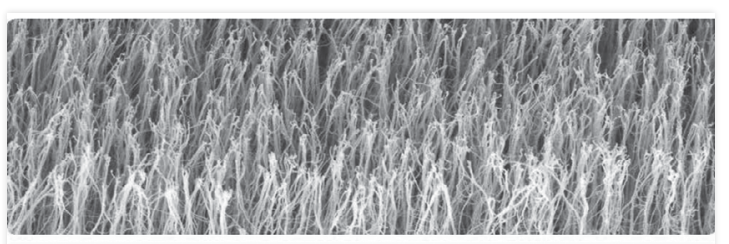

SOCIAL AND ENVIRONMENTAL IMPLICATIONS OF NANOTECHNOLOGY DEVELOPMENT IN ASIA-PACIFIC
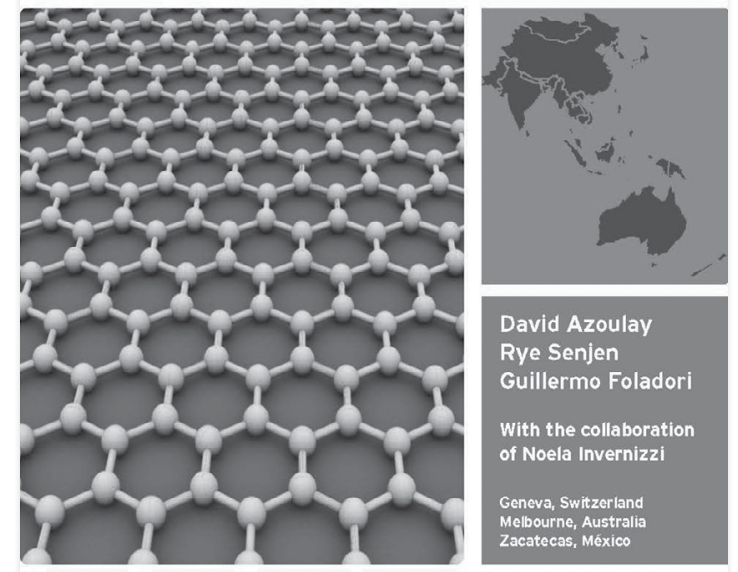

David Azoulay Rye Senjen

Guillermo Foladori

With the collaboration

of Noela Invernizzi

Geneva, Switzer rand

Melbourne, Australia
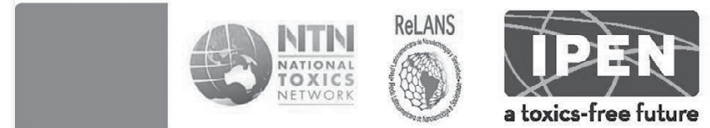